\title{
Courbures et singularités réelles
}

Nicolas Dutertre

Résumé. Soit $f: \mathbb{R}^{n} \rightarrow \mathbb{R}$ un polynôme tel que $f(0)=0$ et tel que 0 soit un point critique isolé de $f$. Etant donnée $f_{t}$ une déformation lisse à un paramètre de $f$, on relie la limite :

$$
\lim _{\varepsilon \rightarrow 0} \lim _{t \rightarrow 0} \int_{C_{t}^{\varepsilon}} k d v_{t}
$$

où $C_{t}^{\varepsilon}=f_{t}^{-1}(0) \cap B_{\varepsilon}^{n}$ est la fibre de Milnor et $k$ la courbure, à des degrés topologiques associés à $f$ et à sa déformation.

Abstract. Let $f: \mathbb{R}^{n} \rightarrow \mathbb{R}$ be a polynomial such that $f(0)=0$ and such that 0 is an isolated critical point of $f$. Given $f_{t}$ a one-parameter smooth deformation of $f$, we relate the limit :

$$
\lim _{\varepsilon \rightarrow 0} \lim _{t \rightarrow 0} \int_{C_{t}^{\varepsilon}} k d v_{t}
$$

where $C_{t}^{\varepsilon}=f_{t}^{-1}(0) \cap B_{\varepsilon}^{n}$ is the Milnor fiber and $k$ the curvature, to topological degrees associated with $f$ and its deformation.

Mathematics Subject Classification (2000). 14P05.

Mots clés. Fibre de Milnor réelle, courbure, degré topologique.

\section{Introduction}

Soit $f: \mathbb{C}^{n} \rightarrow \mathbb{C}$ un polynôme tel que $f(0)=0$ et tel que 0 soit une singularité isolée de $f^{-1}(0)$. Soit $C_{\lambda}^{\varepsilon}=f^{-1}(\lambda) \cap B_{\varepsilon}^{2 n}$ la fibre de Milnor de cette singularité. Langevin [La] étudie le comportement asymptotique de la courbure de LipschitzKilling $K$ de $C_{\lambda}^{\varepsilon}$, définie à l'aide de la métrique induite par celle de $\mathbb{C}^{n} \simeq \mathbb{R}^{2 n}$, quand $\varepsilon$ et $\lambda$ tendent vers 0 . Plus précisément, il obtient la formule suivante :

$$
\lim _{\varepsilon \rightarrow 0} \lim _{\lambda \rightarrow 0} \int_{C_{\lambda}^{\varepsilon}}(-1)^{n-1} K d v=\frac{1}{2} \operatorname{Vol}\left(S^{2 n-2}\right)\left(\mu^{(n)}+\mu^{(n-1)}\right),
$$

où $\mu^{(n)}$ est le nombre de Milnor de $f$ en 0 et $\mu^{(n-1)}$ le nombre de Milnor de la singularité obtenue en coupant $f^{-1}(0)$ par un hyperplan générique. Il montre ainsi que cette limite est finie et qu'elle est liée à des invariants locaux de la singularité.

Nous nous proposons dans cet article de donner une version réelle du résultat 
de Langevin. La situation réelle présente deux différences majeures par rapport à la situation complexe. Tout d'abord, nous avons la possibilité d'étudier deux intégrales de courbure : l'intégrale de la courbure de Gauss et l'intégrale de sa valeur absolue. Cette distinction n'existe pas dans le cas complexe car la courbure de Lipschitz-Killing est de signe constant. D'autre part, il est plus intéressant de travailler sur des fibres du type $C_{t}^{\varepsilon}=f_{t}^{-1}(0) \cap B_{\varepsilon}^{n}$ où $f_{t}$ est une déformation à un paramètre du polynôme $f$. En effet, contrairement au cas complexe où toutes les fibres $f_{t}^{-1}(0) \cap B_{\varepsilon}^{2 n}$ sont homéomorphes à la fibre $f^{-1}(\lambda) \cap B_{\varepsilon}^{2 n}$ et ont l'homotopie d'un bouquet de $\mu^{(n)}$ sphères, le type topologique de la fibre $C_{t}^{\varepsilon}$ dépend de la déformation. Par exemple, quand $f(x, y)=x^{2}-y^{3}$, la fibre $C_{\lambda}^{\varepsilon}$ a une seule composante connexe, qui est homéomorphe à une droite, tandis que la fibre $C_{t}^{\varepsilon}$, où $f_{t}(x, y)=y^{2}-x^{3}-t x$ et $t<0$, a deux composantes, l'une homéomorphe à une droite et l'autre à un cercle. On voit ainsi que la fibre $C_{\lambda}^{\varepsilon}$ n'est pas "maximale", dans le sens où des topologies plus riches que la sienne peuvent apparaître parmi les fibres $C_{t}^{\varepsilon}$, et qu'elle ne donne donc que des informations partielles sur la singularité. Nous sommes amenés à considérer les limites d'intégrales :

$$
\lim _{\varepsilon \rightarrow 0} \lim _{t \rightarrow 0} \int_{C_{t}^{\varepsilon}} k d v_{t} \quad \text { et } \quad \lim _{\varepsilon \rightarrow 0} \lim _{t \rightarrow 0} \int_{C_{t}^{\varepsilon}}|k| d v_{t},
$$

où $k$ désigne la courbure de Gauss et $d v_{t}$ la forme volume de $f_{t}^{-1}(0)$. La deuxième de ces limites a été étudiée par Risler [Ri]. Il montre qu'elle est finie et qu'elle peut se majorer de la manière suivante :

$$
\lim _{\varepsilon \rightarrow 0} \lim _{t \rightarrow 0} \int_{C_{t}^{\varepsilon}}|k| d v_{t} \leq \frac{\operatorname{Vol}\left(S^{n-1}\right)}{\operatorname{Vol}\left(S^{2 n-2}\right)} \lim _{\varepsilon \rightarrow 0} \lim _{t \rightarrow 0} \int_{f_{t, \mathbb{C}}^{-1}(0) \cap B_{\varepsilon}^{2 n}}|K| d v_{t},
$$

où $f_{t, \mathbb{C}}$ est le complexifié de $f_{t}$ et $K$ la courbure de Lipschitz-Killing. Ceci entraîne bien sûr que la première des limites existe et est finie. Une question se pose alors : est-il possible d'avoir des informations plus précises sur ces limites et, en particulier, de les relier à des invariants de la singularité? Dans cette optique, la formule de Gauss-Bonnet, qui exprime la caractéristique d'Euler-Poincaré d'une variété lisse en fonction de l'intégrale de sa courbure, incite plutôt à regarder $\lim _{\varepsilon \rightarrow 0} \lim _{t \rightarrow 0} \int_{C_{t}^{\varepsilon}} k d v_{t}$. C'est ce que nous allons faire dans ce papier.

Soit donc $f: \mathbb{R}^{n} \rightarrow \mathbb{R}$ un polynôme tel que $f(0)=0$ et tel que 0 soit un point critique isolé de $f$. Soit $\left(t, x_{1}, \ldots, x_{n}\right)$ un système de coordonnées de $\mathbb{R}^{1+n}$ et soit $F: \mathbb{R}^{1+n} \rightarrow \mathbb{R}$ un polynôme tel que pour tout $x \in \mathbb{R}^{n}, f(x)=F(0, x)$. La déformation $f_{t}$ est donnée par $f_{t}(x)=F(t, x)$. On associe à $F$ l'application $H: \mathbb{R}^{1+n} \rightarrow \mathbb{R}^{1+n}$ définie par $H=\left(F, \frac{\partial F}{\partial x_{1}}, \ldots, \frac{\partial F}{\partial x_{1}}\right)$ et on suppose que $H$ admet un zéro isolé en 0 . On en déduit immédiatement que $\nabla F$, le vecteur gradient de $F$, admet également un zéro isolé en 0 car, par le lemme du chemin, $(\nabla F)^{-1}(0) \subset$ $F^{-1}(0)$ au voisinage de l'origine. Pour $t \neq 0$ suffisamment petit, la fibre $f_{t}^{-1}(0)=$ $\left\{x \in \mathbb{R}^{n} \mid F(t, x)=0\right\}$ est lisse au voisinage de 0 (Lemme 3.1). La fibre de Milnor correspondant est la variété lisse à bord, éventuellement vide, $C_{t}^{\varepsilon}=f_{t}^{-1}(0) \cap B_{\varepsilon}^{n}$ définie pour $0<|t| \ll \varepsilon \ll 1$. On la munit de la métrique induite par le plongement 
$f_{t}^{-1}(0) \subset \mathbb{R}^{n}$ et on l'oriente par $\nabla f_{t}$, le vecteur gradient de $f_{t}$. On montre alors (Théorème 5.3) :

$\lim _{\varepsilon \rightarrow 0} \lim _{t \rightarrow 0^{+}} \int_{C_{t}^{\varepsilon}} k d v_{t}=-\frac{1}{2} \operatorname{Vol}\left(S^{n-1}\right)\left[\operatorname{deg}_{0} \nabla F+\operatorname{deg}_{0} H\right]+\frac{1}{2} \int_{S^{n-1}} \operatorname{deg}_{0} \nabla\left(f_{\mid P_{v}=0}\right) d v$, $\lim _{\varepsilon \rightarrow 0} \lim _{t \rightarrow 0^{-}} \int_{C_{t}^{\varepsilon}} k d v_{t}=-\frac{1}{2} \operatorname{Vol}\left(S^{n-1}\right)\left[\operatorname{deg}_{0} \nabla F-\operatorname{deg}_{0} H\right]+\frac{1}{2} \int_{S^{n-1}} \operatorname{deg}_{0} \nabla\left(f_{\mid P_{v}=0}\right) d v$, où pour tout $v \in S^{n-1},\left\{P_{v}=0\right\}$ est l'hyperplan $\left\{x \in \mathbb{R}^{n} \mid\langle v, x\rangle=0\right\}(\langle\rangle$, désigne le produit scalaire usuel de $\left.\mathbb{R}^{n}\right)$ et $\operatorname{deg}_{0} H$ (resp. $\operatorname{deg}_{0} \nabla F, \operatorname{deg}_{0} \nabla\left(f_{\mid P_{v}=0}\right)$ ) est le degré topologique de $\frac{H}{\|H\|}$ (resp. $\left.\frac{\nabla F}{\|\nabla F\|}, \frac{\nabla\left(f_{\mid P_{v}=0}\right)}{\left\|\nabla\left(f_{\mid P_{v}=0}\right)\right\|}\right)$ autour d'une petite sphère. On relie ainsi des données géométriques à des données topologiques associées à la singularité et à la déformation. De plus, quand $f$ admet une singularité algébriquement isolée, ces égalités nous permettent de majorer

$$
\left|\lim _{\varepsilon \rightarrow 0} \lim _{t \rightarrow 0^{ \pm}} \int_{C_{t}^{\varepsilon}} k d v_{t}\right|
$$

en fonction de $\operatorname{deg}_{0} \nabla F, \operatorname{deg}_{0} H$ et $\mu^{(n-1)}\left(f_{\mathbb{C}}\right)$, où $f_{\mathbb{C}}$ est le complexifié de $f$ (Corollaire 5.5). On termine notre étude en expliquant comment, pour le cas des courbes planes, le terme $\int_{S^{n-1}} \operatorname{deg}_{0} \nabla\left(f_{\mid P_{v}=0}\right) d v$ s'exprime simplement en fonction de $f$ (Théorème 6.1).

Pour prouver son résultat, Langevin utilise une formule d'échange qui permet de calculer l'intégrale de la courbure de Lipschitz-Killing en fonction du nombre de points critiques de la projection orthogonale de la fibre $C_{\lambda}^{\varepsilon}$ sur une droite complexe générique. Ce nombre est le nombre de points d'intersection d'une courbe polaire générique avec la fibre et il tend vers $\mu^{(n)}+\mu^{(n-1)}$ d'après Teissier [Te]. Risler reprend cette démarche en majorant le nombre de points critiques de la projection orthogonale de $C_{t}^{\varepsilon}$ sur une droite réelle générique par le nombre de points critiques de la projection de la fibre complexifiée sur la droite complexifiée et en appliquant des formules d'échange. Notre démonstration repose également sur l'étude de ces points critiques et sur une formule d'échange. Cependant nous n'évaluons pas le nombre de ces points critiques mais la somme, prise sur l'ensemble de ces points, des indices de l'application de Gauss. Cette somme est ensuite reliée via la théorie de Morse à la caractéristique d'Euler-Poincaré de la fibre, puis à des degrés topologiques par une formule due à Fukui. On applique alors une formule d'échange pour conclure.

Le papier est organisé comme suit : la Section 2 est un calcul préliminaire d'indice. On rappelle la formule de Fukui dans la Section 3. La Section 4 est consacrée aux courbes polaires. Le résultat principal est prouvé dans la Section 5. Finalement on étudie le cas des courbes planes dans la Section 6 .

Des exemples explicites sont donnés dans les Sections 5 et 6 . Pour le calcul des degrés topologiques, nous utilisons un programme dû à Lecki et basé sur la formule d'Eisenbud-Levine [EL]. L'algorithme employé est détaillé dans [LS]. Le programme est disponible auprès de Z. Szafraniec. 
L'auteur remercie vivement M. Nicolau et J.-J. Risler de lui avoir suggéré ce travail.

Ce travail a été financé par la bourse Marie Curie HPMFCT-2000-00645 du programme de la Communauté Europénne Improving Human Research Potential and the Socio-economic Knowledge Base. Il a été réalisé au Centre de Recerca Matemàtica de Barcelone. L'auteur tient à remercier cette institution pour son hospitalité.

\section{Un calcul d'indice}

On exprime le degré de l'application de Gauss en un point d'une hypersurface lisse en fonction de l'indice de Morse d'une fonction hauteur.

Soit $f: \mathbb{R}^{n} \rightarrow \mathbb{R}$ une fonction de classe $C^{2}$ telle que $W=f^{-1}(0)$ soit une hypersurface lisse, orientée par $\nabla f$. Soit

$$
\begin{aligned}
\gamma: W & \rightarrow S^{n-1} \\
x & \mapsto \frac{\nabla f(x)}{\|\nabla f(x)\|}
\end{aligned}
$$

l'application de Gauss et, pour tout $v \in S^{n-1}$, soit $P_{v}$ la fonction hauteur définie $\operatorname{par} P_{v}(x)=\langle v, x\rangle$.

Lemme 2.1. Un point $p$ de $W$ est un point critique de $P_{v \mid W}$ si et seulement si $\gamma(p)= \pm v$.

Preuve. Clair.

Dans la suite, on fixe $v=(1,0, \ldots, 0)$ et on considère un point critique $p$ de $x_{1 \mid W}$. Dans ce cas $\frac{\partial f}{\partial x_{1}}(p) \neq 0$ et l'on peut paramétrer $W$ au voisinage de $p$ en fonction de $x_{2}, \ldots, x_{n}$. Il existe une bijection $\theta: \mathbb{R}^{n-1} \rightarrow W$, de classe $C^{2}$, d'un voisinage de $p^{\prime}=\left(x_{2}(p), \ldots, x_{n}(p)\right)$ sur un voisinage de $p$, de la forme $\theta\left(x^{\prime}\right)=\left(\varphi\left(x^{\prime}\right), x^{\prime}\right)$, où $x^{\prime}=\left(x_{2}, \ldots, x_{n}\right)$.

Soit $\operatorname{deg}\left(\theta, p^{\prime}\right)$ le degré de $\theta$ en $p^{\prime}$; il vaut +1 si $\theta$ conserve l'orientation et -1 sinon.

Lemme 2.2. $\operatorname{deg}\left(\theta, p^{\prime}\right)=$ signe $\frac{\partial f}{\partial x_{1}}(p)$.

Preuve. Soit $\left(e_{2}, \ldots, e_{n}\right)$ la base canonique directe de $\mathbb{R}^{n-1}$. Une base de l'espace tangent à $W$ en $p$ est donnée par $\left(d_{p^{\prime}} \theta\left(e_{2}\right), \ldots, d_{p^{\prime}} \theta\left(e_{n}\right)\right), d_{p^{\prime}} \theta$ étant la différentielle de $\theta$ en $p^{\prime}$, et $\theta$ conserve l'orientation en $p^{\prime}$ si et seulement si $\left(\nabla f(p), d_{p^{\prime}} \theta\left(e_{2}\right), \ldots, d_{p^{\prime}} \theta\left(e_{n}\right)\right)$ est une base directe de $\mathbb{R}^{n}$. Notons $\mathcal{B}$ cette base 
et soit $M$ la matrice de $\mathcal{B}$ dans la base canonique $\left(e_{1}, \ldots, e_{n}\right)$ de $\mathbb{R}^{n}$. On a

$$
M=\left(\begin{array}{cccc}
\frac{\partial f}{\partial x_{1}}(p) & \frac{\partial \varphi}{\partial x_{2}}\left(p^{\prime}\right) & \cdots & \frac{\partial \varphi}{\partial x_{n}}\left(p^{\prime}\right) \\
\frac{\partial f}{\partial x_{2}}(p) & 1 & \cdots & 0 \\
\vdots & \vdots & \ddots & \vdots \\
\frac{\partial f}{\partial x_{n}}(p) & 0 & \cdots & 1
\end{array}\right)
$$

Comme $f\left(\varphi\left(x^{\prime}\right), x^{\prime}\right) \equiv 0$, pour tout $i \in\{2, \ldots, n\}$

$$
\frac{\partial f}{\partial x_{1}}\left(\theta\left(x^{\prime}\right)\right) \cdot \frac{\partial \varphi}{\partial x_{i}}\left(x^{\prime}\right)+\frac{\partial f}{\partial x_{i}}\left(\theta\left(x^{\prime}\right)\right)=0 .
$$

Appliquée en $p^{\prime}$, cette égalité permet de montrer que

$$
\text { signe } \operatorname{det}(M)=\text { signe } \frac{\partial f}{\partial x_{1}}(p) \text {. }
$$

Soit $\operatorname{deg}(\gamma, p)$ le degré de $\gamma$ en $p$.

Lemme 2.3. Le point critique $p$ de $x_{1 \mid W}$ est non-dégénéré si et seulement si $p$ est un point régulier de $\gamma$. Dans ce cas,

$$
\operatorname{deg}(\gamma, p)=(-1)^{n-1} \cdot \operatorname{signe}\left(\frac{\partial f}{\partial x_{1}}\right)^{n-1}(p) \cdot(-1)^{\lambda}
$$

où $\lambda$ est l'indice de Morse de $x_{1 \mid W}$ en $p$.

Preuve. Soit $\beta: \mathbb{R}^{n-1} \rightarrow S^{n-1}$ une paramétrisation de $S^{n-1}$ au voisinage de $\gamma(p)$. Le lemme précédent pour le cas de la fonction $x_{1}^{2}+\cdots+x_{n}^{2}-1$ donne

$$
\operatorname{deg}\left(\beta, \beta^{-1}(\gamma(p))=\text { signe } x_{1}(\gamma(p))=\operatorname{signe} \frac{\partial f}{\partial x_{1}}(p) .\right.
$$

On considère ensuite $\tilde{\gamma}:\left(\mathbb{R}^{n-1}, p^{\prime}\right) \rightarrow\left(\mathbb{R}^{n-1}, 0\right)$ définie par $\tilde{\gamma}\left(x^{\prime}\right)=\beta^{-1} \circ \gamma \circ \theta\left(x^{\prime}\right)$. On voit que $p$ est un point régulier de $\gamma$ si et seulement si $p^{\prime}$ est un point régulier de $\tilde{\gamma}$ et que $\operatorname{deg}(\gamma, p)=\operatorname{deg}\left(\tilde{\gamma}, p^{\prime}\right)$ puisque $\operatorname{deg}\left(\beta^{-1}, \gamma(p)\right)=\operatorname{deg}\left(\beta, \beta^{-1}(\gamma(p))\right)$. Soit pour tout $i \in\{2, \ldots, n\}, \tilde{\gamma}_{i}$ la $i$-ème coordonnée de $\tilde{\gamma}$. Si $p^{\prime}$ est un point régulier de $\tilde{\gamma}$ alors

$$
\operatorname{deg}\left(\tilde{\gamma}, p^{\prime}\right)=\operatorname{signe} \operatorname{det}\left(\frac{\partial \tilde{\gamma}_{i}}{\partial x_{j}}\right)_{2 \leq i, j \leq n}\left(p^{\prime}\right) .
$$

En dérivant l'égalité

$$
\tilde{\gamma}_{i}\left(x^{\prime}\right)=\frac{\frac{\partial f}{\partial x_{i}}\left(\theta\left(x^{\prime}\right)\right)}{\left\|\nabla f\left(\theta\left(x^{\prime}\right)\right)\right\|}
$$

et en utilisant le fait que $\frac{\partial f}{\partial x_{i}}(p)=0$, on trouve que pour tout $(i, j) \in\{2, \ldots, n\}^{2}$ :

$$
\frac{\partial \tilde{\gamma}_{i}}{\partial x_{j}}\left(p^{\prime}\right)=\frac{1}{\|\nabla(f(p))\|}\left(\frac{\partial^{2} f}{\partial x_{i} \partial x_{1}}(p) \cdot \frac{\partial \varphi}{\partial x_{j}}\left(p^{\prime}\right)+\frac{\partial^{2} f}{\partial x_{i} \partial x_{j}}(p)\right) .
$$


D'après $(1), \frac{\partial \varphi}{\partial x_{j}}\left(p^{\prime}\right)$ est nul si bien que

$$
\operatorname{det}\left(\frac{\partial \tilde{\gamma}_{i}}{\partial x_{j}}\left(p^{\prime}\right)\right)_{2 \leq i, j \leq n}=\frac{1}{\|\nabla f(p)\|^{n-1}} \cdot \operatorname{det}\left(\frac{\partial^{2} f}{\partial x_{i} \partial x_{j}}(p)\right)_{2 \leq i, j \leq n} .
$$

Il faut ensuite dériver (1) par rapport à $x_{j}$ et utiliser l'annulation en $p^{\prime}$ des dérivées partielles de $\varphi$ pour obtenir

et pouvoir ainsi conclure.

$$
\frac{\partial^{2} f}{\partial x_{i} \partial x_{j}}(p)=-\frac{\partial f}{\partial x_{1}}(p) \cdot \frac{\partial^{2} \varphi}{\partial x_{i} \partial x_{j}}\left(p^{\prime}\right)
$$

\section{Fibre de Milnor et degrés topologiques}

On rappelle que $H$ est donnée par $H=\left(F, \frac{\partial F}{\partial x_{1}}, \ldots, \frac{\partial F}{\partial x_{n}}\right)$ et que $H$ et $\nabla F$ admettent un zéro isolé en 0 .

Dans cette partie, on montre que la fibre de Milnor est lisse puis on rappelle la formule de Fukui [Fu1], ingrédient essentiel de notre preuve, qui relie $\chi\left(C_{t}^{\varepsilon}\right)$ et $\chi\left(\left\{f_{t} \geq 0\right\} \cap B_{\varepsilon}^{n}\right)-\chi\left(\left\{f_{t} \leq 0\right\} \cap B_{\varepsilon}^{n}\right)$ aux degrés topologiques en 0 de $H, \nabla F$ et $\nabla f$.

Lemme 3.1. Pour $t \neq 0$ suffisamment petit, la fibre $f_{t}^{-1}(0)$ n'admet pas de singularités au voisinage de 0 .

Preuve. Un point $p$ de $f_{t^{\prime}}^{-1}(0)$ est un point singulier de $f_{t^{\prime}}^{-1}(0)$ si et seulement si $\left(t^{\prime}, p\right)$ est un point singulier de $\left\{t=t^{\prime}\right\} \cap F^{-1}(0)$. Comme $(0,0)$ est isolé dans $H^{-1}(0)$, il n'existe pas de tel point autre que $(0,0)$ au voisinage de l'origine.

Théorème 3.2. Soit $t$ tel que $0<|t| \ll \varepsilon \ll 1$, si $n$ est pair alors

- $\chi\left(C_{t}^{\varepsilon}\right)=1-\operatorname{deg}_{0} \nabla f$

- $\chi\left(\left\{f_{t} \geq 0\right\} \cap B_{\varepsilon}\right)-\chi\left(\left\{f_{t} \leq 0\right\} \cap B_{\varepsilon}\right)=\operatorname{deg}_{0} \nabla F+\operatorname{signe}(t) \cdot \operatorname{deg}_{0} H$.

Si $n$ est impair alors

- $\chi\left(C_{t}^{\varepsilon}\right)=1-\operatorname{deg}_{0} \nabla F-\operatorname{signe}(t) \cdot \operatorname{deg}_{0} H$,

- $\chi\left(\left\{f_{t} \geq 0\right\} \cap B_{\varepsilon}\right)-\chi\left(\left\{f_{t} \leq 0\right\} \cap B_{\varepsilon}\right)=\operatorname{deg}_{0} \nabla f$.

Preuve. On montre le cas $n$ pair. Fukui ([Fu1], p52-53) prouve que

$$
\begin{aligned}
& \chi\left(\left\{f_{t} \geq 0\right\} \cap B_{\varepsilon}\right)=1+\frac{1}{2}\left(\operatorname{signe}(t) \cdot \operatorname{deg}_{0} H+\operatorname{deg}_{0} \nabla F-\operatorname{deg}_{0} \nabla f\right), \\
& \chi\left(\left\{f_{t} \leq 0\right\} \cap B_{\varepsilon}\right)=1-\frac{1}{2}\left(\operatorname{signe}(t) \cdot \operatorname{deg}_{0} H+\operatorname{deg}_{0} \nabla F+\operatorname{deg}_{0} \nabla f\right) .
\end{aligned}
$$

On en déduit la formule pour $\chi\left(\left\{f_{t} \geq 0\right\} \cap B_{\varepsilon}\right)-\chi\left(\left\{f_{t} \leq 0\right\} \cap B_{\varepsilon}\right)$. La suite de Mayer-Vietoris donne

$$
\chi\left(B_{\varepsilon}\right)=\chi\left(\left\{f_{t} \geq 0\right\} \cap B_{\varepsilon}\right)+\chi\left(\left\{f_{t} \leq 0\right\} \cap B_{\varepsilon}\right)-\chi\left(\left\{f_{t}=0\right\} \cap B_{\varepsilon}\right) .
$$


Comme $B_{\varepsilon}$ est contractile, on obtient aisément la formule pour $\chi\left(C_{t}^{\varepsilon}\right)$.

Remarque 3.3. Quand $F(t, x)=f(x)-t, \operatorname{deg}_{0} \nabla F=0$ et $\operatorname{deg}_{0} H=-\operatorname{deg}_{0} \nabla f$. On retrouve la formule de Khimshiashvili [Kh] (voir aussi [Ar], [Fu2], [Wa]) :

$$
\begin{gathered}
\chi\left(f^{-1}(\lambda) \cap B_{\varepsilon}^{n}\right)=1-\operatorname{deg}_{0} \nabla f \text { si } n \text { est pair, } \\
\chi\left(f^{-1}(\lambda) \cap B_{\varepsilon}^{n}\right)=1+\operatorname{signe}(\lambda) \cdot \operatorname{deg}_{0} \nabla f \text { si } n \text { est impair. }
\end{gathered}
$$

De plus,

$$
\begin{gathered}
\chi\left(\{f \geq \lambda\} \cap B_{\varepsilon}\right)-\chi\left(\{f \leq \lambda\} \cap B_{\varepsilon}\right)=\operatorname{signe}(\lambda) \cdot \operatorname{deg}_{0} \nabla f \text { si } n \text { est pair } \\
\chi\left(\{f \geq \lambda\} \cap B_{\varepsilon}\right)-\chi\left(\{f \leq \lambda\} \cap B_{\varepsilon}\right)=\operatorname{deg}_{0} \nabla f \text { si } n \text { est impair. }
\end{gathered}
$$

\section{Fonctions hauteurs et courbes polaires}

Pour tout $v \in S^{n-1}$, soit $P_{v}$ la fonction hauteur définie par $P_{v}(x)=\langle v, x\rangle$. On montre que génériquement 0 est un point critique isolé de $f_{\mid P_{v}=0}$ et $P_{v \mid C_{t}^{\varepsilon}}$ est une fonction de Morse pour $t$ et $\varepsilon$ suffisamment petits. Ce dernier point s'établit en étudiant les propriétés des courbes polaires génériques.

Proposition 4.1. Il existe un ouvert dense semi-algébrique $O_{1}$ de $S^{n-1}$ tel que pour tout $v \in O_{1}, f:\left\{P_{v}=0\right\} \rightarrow \mathbb{R}$ admette un point critique isolé à l'origine.

Preuve. Soit $g: \mathbb{R}^{n} \times \mathbb{R}^{n} \rightarrow \mathbb{R}$ définie par $g(x, y)=\langle x, y\rangle$ et soit

$$
M=\left\{(x, y) \in \mathbb{R}^{n} \times \mathbb{R}^{n} \mid f(x)=0 \text { et } g(x, y)=0\right\} .
$$

Pour tout $(x, y) \in X=M \backslash\left(\{0\} \times \mathbb{R}^{n}\right), x$ proche de l'origine, on voit que $\operatorname{rang}(\nabla f, \nabla g)=2$ en $(x, y)$ car $x \notin \nabla f^{-1}(0)$. On en déduit que $X$ est une variété de Nash de dimension $2 n-2$. Soit

$$
\begin{aligned}
\Pi_{y}: X & \rightarrow \mathbb{R}^{n} \\
(x, y) & \mapsto y .
\end{aligned}
$$

Le théorème de Bertini-Sard pour les variétés de Nash (voir [BCR], 9.5.2) entraîne que l'ensemble $\Sigma$ des valeurs critiques de $\Pi_{y}$ est un semi-algébrique de dimension strictement inférieure à $n$. Pour tout $v \notin \Sigma$ et pour tout $x \in \Pi_{y}^{-1}(v), x \neq 0$ proche de l'origine, on a $\operatorname{rang}(\nabla f, v)=2$. On prend alors $O_{1}=S^{n-1} \backslash \Sigma$.

Remarque 4.2. Lorsque $f$ admet une singularité algébriquement isolée à l'origine, la même démonstration appliquée au complexifié $f_{\mathbb{C}}$ de $f$ montre que l'on peut choisir $O_{1}$ de sorte que pour tout $v \in O_{1}, f:\left\{P_{v}=0\right\} \rightarrow \mathbb{R}$ admette une singularité algébriquement isolée à l'origine. Dans ce cas, le nombre de Milnor du complexifié de $f:\left\{P_{v}=0\right\} \rightarrow \mathbb{R}$ est égal au nombre de Milnor d'une coupe hyperplane générique de $f_{\mathbb{C}}^{-1}(0)$. 
Dans ce qui suit, on désignera aussi par $P_{v}$ la fonction $\mathbb{R}^{n+1} \rightarrow \mathbb{R},(t, x) \mapsto$ $\langle v, x\rangle$.

Proposition 4.3. Il existe un ouvert semi-algébrique dense $\mathrm{O}_{2}$ de $\mathrm{S}^{n-1}$ tel que pour tout $v \in O_{2}, F^{-1}(0) \cap\left\{P_{v}=0\right\} \backslash\{0\}$ soit vide ou bien lisse de codimension 2 .

Preuve. Soit $h: \mathbb{R}^{1+n} \times \mathbb{R}^{n} \rightarrow \mathbb{R}$ définie par $h(t, x, y)=\langle x, y\rangle$ et soit

$$
N=\left\{(t, x, y) \in \mathbb{R}^{1+n} \times \mathbb{R}^{n} \mid F(t, x)=0 \text { et } h(t, x, y)=0\right\} .
$$

Pour tout $(t, x, y) \in N \backslash\left\{x_{1}=\ldots=x_{n}=0\right\},(t, x)$ proche de $(0,0), \operatorname{rang}(\nabla F, \nabla h)$ $=2 \operatorname{car}(t, x) \notin(\nabla F)^{-1}(0)$. Comme pour la Proposition 4.1, on peut choisir un ouvert semi-algébrique $O^{\prime}$ de $S^{n-1}$ tel que pour tout $v \in O^{\prime}, \operatorname{rang}(\nabla F, v)=2$ sur $\{F=0\} \cap\left\{P_{v}=0\right\} \backslash\left\{x_{1}=\ldots=x_{n}=0\right\}$.

On a montré que génériquement $F^{-1}(0) \cap\left\{P_{v}=0\right\} \backslash\{0\}$ est vide ou lisse de codimension 2 en dehors de $\left\{x_{1}=\ldots=x_{n}=0\right\}$. Il reste à étudier la situation quand $x_{1}=\ldots=x_{n}=0$. On va voir qu'en fait on peut choisir génériquement $v$ de sorte que $\{F=0\} \cap\left\{P_{v}=0\right\}$ n'intersecte pas $\left\{x_{1}=\ldots=x_{n}=0\right\}$ en dehors de l'origine.

Soit, pour tout $(i, j) \in\{1, \ldots, n\}^{2}, m_{i j}=\left|\begin{array}{cc}\frac{\partial F}{\partial x_{i}} & \frac{\partial F}{\partial x_{j}} \\ y_{i} & y_{j}\end{array}\right|$ et soit

$$
\begin{aligned}
N^{\prime}=\left\{(t, x, y) \in \mathbb{R}^{1+n} \times \mathbb{R} \mid\right. & x_{1}=0, \ldots, x_{n}=0, F(t, x)=0 \\
& \text { et pour tout } \left.(i, j) \in\{1, \ldots, n\}^{2}, m_{i j}=0\right\} .
\end{aligned}
$$

L'ensemble $Y=N^{\prime} \backslash\left(\{(0,0)\} \times \mathbb{R}^{n}\right)$ est une variété analytique lisse de dimension 1 au voisinage de $(0,0,0)$. Pour voir cela, considérons un point $(t, x, y)$ de $Y$. On peut supposer qu'en ce point $\frac{\partial F}{\partial x_{1}}(t, x) \neq 0$, ce qui entraîne qu'autour de $(t, x, y) Y$ est défini par l'annulation des fonctions $x_{1}, \ldots, x_{n}, F, m_{12}, \ldots, m_{1 n}$. Un simple calcul de déterminant montre que les vecteurs gradients de ces fonctions sont linéairement indépendants.

En utilisant la projection $\pi_{y}: Y \rightarrow \mathbb{R}^{n},(t, x, y) \mapsto y$, on trouve alors un ouvert semi-algébrique $O$ " de $S^{n-1}$ tel que pour tout $v \in O$ ", $F^{-1}(0) \cap\left\{x_{1}=\ldots=x_{n}=\right.$ $0\} \cap\{\operatorname{rang}(\nabla F, v)<2\}$ soit de dimension au plus zéro au voisinage de $(0,0)$. Il suffit de prendre $\mathrm{O}_{2}=\mathrm{O}^{\prime} \cap \mathrm{O}^{\prime \prime}$.

Proposition 4.4. Il existe un ouvert dense semi-algébrique $\mathrm{O}_{3} \subset \mathrm{O}_{2} \subset S^{n-1}$ tel que pour tout $v \in O_{3}, t:\left\{P_{v}=0\right\} \cap\{F=0\} \rightarrow \mathbb{R}$ n'admette pas de points critiques en dehors de 0.

Preuve. Soit

$$
Q=\left\{(t, x, y) \in \mathbb{R}^{1+n} \times \mathbb{R} \mid t=0, F(t, x)=0 \text { et } h(t, x, y)=0\right\} .
$$

L'ensemble $Q \backslash\left\{(0,0) \times \mathbb{R}^{n}\right\}$ est une variété analytique lisse de codimension 3 et, comme dans les propositions précédentes, on peut trouver $V$ tel que pour tout $v \in V,\{t=0\},\{F=0\}$ et $\left\{P_{v}=0\right\}$ s'intersectent transversalement en dehors 
de $(0,0)$. On pose $O_{3}=O_{2} \cap V$. Pour tout $v \in O_{3},\{F=0\} \cap\left\{P_{v}=0\right\}$ est soit lisse de codimension 2 soit vide en dehors de $(0,0)$ et une application du lemme $\mathrm{du}$ chemin montre que les points critiques de $t_{\mid\left\{P_{v}=F_{v}=0\right\} \backslash\{0\}}$ ne peuvent se situer que sur le niveau $\{t=0\}$.

Pour tout $v \in S^{n-1}$, on définit la courbe polaire :

$$
L_{v}=\left\{(t, x) \in \mathbb{R}^{1+n} \mid F(t, x)=0 \text { et } \operatorname{rang}(\nabla t, \nabla F, v)<3\right\} .
$$

Il est clair que $0 \in L_{v}$.

Proposition 4.5. Il existe un ouvert semi-algébrique $O_{4}$ de $S^{n-1}$ tel que pour tout $v \in O_{4}, L_{v} \backslash\{(0,0)\}$ est soit vide, soit une courbe au voisinage de $(0,0)$.

Preuve. Soit

$$
M=\left\{(t, x, y) \in \mathbb{R}^{1+n} \times \mathbb{R}^{n} \mid F(t, x)=0 \text { et } m_{i j}(x)=0\right\} .
$$

Si un point $(t, x, y) \in M \backslash\left\{(0,0) \times \mathbb{R}^{n}\right\}$ est tel que $\frac{\partial F}{\partial x_{1}}(t, x) \neq 0$ alors $M \backslash\{(0,0) \times$ $\left.\mathbb{R}^{n}\right\}$ est définie dans un voisinage de $(t, x, y)$ par l'annulation de $F, m_{12}, \ldots, m_{1 n}$, dont les vecteurs gradients respectifs sont indépendants. Ainsi $M \backslash\left\{(0,0) \times \mathbb{R}^{n}\right\}$ est une variété analytique lisse de dimension $n+1$. On conclut en utilisant la projection $\pi_{y}$.

On pose $U=O_{1} \cap O_{3} \cap O_{4}$.

Lemme 4.6. Pour tout $v \in U, L_{v} \cap\{t=0\}=\{0\}$ et $L_{v} \cap\left\{P_{v}=0\right\}=\{0\}$ au voisinage de 0 .

Preuve. On écrit $v=\left(v_{1}, \ldots, v_{n}\right)$. Supposons qu'il existe un $\operatorname{arc} \Gamma$ de $L_{v}$ inclus dans $\{t=0\}$ et considérons une paramétrisation analytique $\alpha:[0, \nu[\rightarrow \Gamma, \alpha(0)=0$, de cet arc. Les dérivées des fonctions $t \circ \alpha$ et $F \circ \alpha$ étant identiquement nulles, on trouve que

$$
\sum_{i=1}^{n} \frac{\partial F}{\partial x_{i}}(\alpha(s)) \cdot \frac{\partial\left(x_{i} \circ \alpha\right)}{\partial s}(s) \equiv 0 .
$$

Le long de $\Gamma$, les vecteurs $\left(\frac{\partial F}{\partial x_{1}}, \ldots, \frac{\partial F}{\partial x_{n}}\right)$ et $\left(v_{1}, \ldots, v_{n}\right)$ sont dépendants et le premier ne s'annule pas. Ceci entraîne que

$$
\sum_{i=1}^{n} v_{i} \cdot \frac{\partial\left(x_{i} \circ \alpha\right)}{\partial s}(s) \equiv 0,
$$

et que $P_{v}(\alpha(s)) \equiv 0$. Cette dernière relation contredit la Proposition 4.4. La seconde égalité du lemme est une conséquence immédiate de la Proposition 4.4.

Lemme 4.7. Pour tout $v \in U, L_{v}$ est définie par l'annulation de $n$ polynômes dont les vecteurs gradients forment un système libre. 
Preuve. On suppose que $v_{1} \neq 0$. La courbe $L_{v}$ est alors définie par l'annulation de $F$ et des mineurs $m_{1 i}, i \in\{2, \ldots, n\}$. Soit $(t, x)$ un point de $L_{v} \backslash\{(0,0)\}$. On voit facilement que $\frac{\partial F}{\partial x_{1}}(t, x) \neq 0$, sinon $H(t, x)=0$. D'après la Proposition 4.5, ceci entraîne que $\operatorname{rang}\left(\nabla F, \nabla m_{12}, \ldots, \nabla m_{1 n}\right)=n$.

Lemme 4.8. Pour tout $v \in U$, il existe $\delta^{\prime}$ tel que $0<\delta^{\prime} \ll \varepsilon$ et tel que pour tout $\delta$ avec $0<|\delta| \leq \delta^{\prime}$, les points critiques de $P_{v_{\mid F^{-1}}(0) \cap\{t=\delta\} \cap B_{\varepsilon}^{n+1}}$ situés à l'intérieur de $F^{-1}(0) \cap\{t=\delta\} \cap B_{\varepsilon}^{n+1}$ soient de Morse. De plus, ils ne sont pas sur le niveau $\left\{P_{v}=0\right\}$.

Preuve. On suppose que $v_{1} \neq 0$. Soit $\Gamma$ un arc de $L_{v}$ paramétré analytiquement par $\alpha:[0, \nu[\rightarrow \Gamma, \alpha(0)=0$. D'après le Lemme 4.6, on peut choisir $\nu$ de sorte que $t(\alpha(0))=0$ et $t(\alpha(s)) \neq 0$ sur $] 0, \nu[$. La fonction $t \circ \alpha$ étant analytique est strictement monotone au voisinage de $(0,0)$, ce qui implique que pour tout $s \in] 0, \nu\left[,\left\langle\nabla t(\alpha(s)), \alpha^{\prime}(s)\right\rangle \neq 0\right.$. Les vecteurs $\nabla t(\alpha(s)), \nabla F(\alpha(s)), \nabla m_{12}(\alpha(s))$, $\ldots, \nabla m_{1 n}(\alpha(s))$ sont donc linéairement indépendants puisque $\nabla F(\alpha(s))$ et les $\nabla m_{1 i}(\alpha(s))$ sont orthogonaux à $\alpha^{\prime}(s)$. D'après [Sz], Lemma 3.2, ceci équivaut à ce que $P_{v}: t^{-1}(t(\alpha(s))) \cap F^{-1}(0) \rightarrow \mathbb{R}$ admette un point critique non-dégénéré en $\alpha(s)$. On choisit alors $\delta^{\prime}>0$ tel que $\delta^{\prime}$ soit inférieur au minimum, pris sur l'ensemble des arcs de $L_{v}$, des $|t(\alpha(\nu))|$.

Corollaire 4.9. Pour tout $v \in U$, il existe $t^{\prime}, 0<t^{\prime} \ll \varepsilon$, tel que pour tout $t$ avec $0<|t| \leq t^{\prime}$, les points critiques de $P_{v \mid C_{t}^{\varepsilon}}$ situés à l'intérieur de $C_{t}^{\varepsilon}$ soient de Morse. De plus, ils ne sont pas sur le niveau $\left\{P_{v}=0\right\}$.

Preuve. Il suffit de voir que $p$ est un point critique de Morse de $P_{v_{\mid f}^{-1}(0)}$ si et seulement si $(\delta, p)$ est un point critique de Morse de $P_{v \mid\{t=\delta\} \cap\{F=0\}}$.

\section{Résultat principal}

Soit $\gamma_{t, \varepsilon}$ l'application de Gauss définie par :

$$
\begin{aligned}
\gamma_{t, \varepsilon}: C_{t}^{\varepsilon} & \rightarrow S^{n-1} \\
x & \mapsto \frac{\nabla f_{t}(x)}{\left\|\nabla f_{t}(x)\right\|} .
\end{aligned}
$$

On choisit $v \in U$ et on appelle $I(t, \varepsilon, v)=\sum_{j} \operatorname{deg}\left(\gamma_{t, \varepsilon}, q_{j}\right)$ où $\left\{q_{j}\right\}=\gamma_{t, \varepsilon}^{-1}(v)$ et $\operatorname{deg}\left(\gamma_{t, \varepsilon}, q_{j}\right)$ est le degré de $\gamma_{t, \varepsilon}$ en $q_{j}$. Notre premier objectif (Théorème 5.2) est de lier

$$
\lim _{\varepsilon \rightarrow 0} \lim _{t \rightarrow 0} I(t, \varepsilon, v)+I(t, \varepsilon,-v)
$$

aux degrés topologiques de $H, \nabla F$ et $\nabla\left(f_{\mid P_{v}=0}\right)$. Pour alléger l'écriture, on pose $\left\{p_{i}\right\}=\gamma_{t, \varepsilon}^{-1}(+v) \cup \gamma_{t, \varepsilon}^{-1}(-v)$. D'après le Lemme 2.1, les points $p_{i}$ sont exactement les points critiques de $P_{v \mid C_{t}^{\varepsilon}}$. De plus, le Corollaire 4.9 implique que, pour $0<$ 
$|t| \ll \varepsilon \ll 1$, ces points critiques sont non-dégénérés et qu'ainsi ils sont en nombre fini. On appelle $\lambda_{i}$ l'indice de Morse de $p_{i}$.

Lemme 5.1. Pour $0<|t| \ll \varepsilon \ll 1$, si n est impair

$$
I(t, \varepsilon, v)+I(t, \varepsilon,-v)=\chi\left(C_{t}^{\varepsilon}\right)-\chi\left(C_{t}^{\varepsilon} \cap\left\{P_{v}=0\right\}\right) .
$$

Si $n$ est pair,

$$
\begin{aligned}
& I(t, \varepsilon, v)+I(t, \varepsilon,-v)=-\left\{\chi\left(\left\{f_{t} \geq 0\right\} \cap B_{\varepsilon}\right)-\chi\left(\left\{f_{t} \leq 0\right\} \cap B_{\varepsilon}\right)\right\} \\
& \quad+\left\{\chi\left(\left\{f_{t} \geq 0\right\} \cap B_{\varepsilon} \cap\left\{P_{v}=0\right\}\right)-\chi\left(\left\{f_{t} \leq 0\right\} \cap B_{\varepsilon} \cap\left\{P_{v}=0\right\}\right)\right\} .
\end{aligned}
$$

Preuve. On donne la preuve pour $v=(1,0 \ldots, 0)$. D'après la Corollaire 4.9 , pour $t$ et $\varepsilon$ suffisamment petits, les points critiques de $x_{1 \mid C_{t}^{\varepsilon}}$ ne sont pas sur le niveau $\left\{x_{1}=0\right\}$ et donc $C_{t}^{\varepsilon} \cap\left\{x_{1}=0\right\}$ est une variété à bord de dimension $n-2$. Si $n$ est impair, par la théorie de Morse, on a

$$
\begin{aligned}
& \chi\left(C_{t}^{\varepsilon} \cap\left\{x_{1} \geq 0\right\}, C_{t}^{\varepsilon} \cap\left\{x_{1}=0\right\}\right)=\sum_{x_{1}\left(p_{i}\right)>0}(-1)^{\lambda_{i}}, \\
& \chi\left(C_{t}^{\varepsilon} \cap\left\{x_{1} \leq 0\right\}, C_{t}^{\varepsilon} \cap\left\{x_{1}=0\right\}\right)=\sum_{x_{1}\left(p_{i}\right)<0}(-1)^{\lambda_{i}} .
\end{aligned}
$$

Le Lemme 2.3 appliqué à $\gamma_{t, \varepsilon}$ et au point $p_{i}$ donne

$$
\operatorname{deg}\left(\gamma_{t, \varepsilon}\right)=(-1)^{n-1} \cdot\left(\frac{\partial f_{t}}{\partial x_{1}}\left(p_{i}\right)\right)^{n-1} \cdot(-1)^{\lambda_{i}} .
$$

En additionnant (1) et (2), en utilisant la suite de Mayer-Vietoris et la relation (3), on obtient

$$
\chi\left(C_{t}^{\varepsilon}\right)-\chi\left(C_{t}^{\varepsilon} \cap\left\{x_{1}=0\right\}\right)=I(t, \varepsilon, v)+I(t, \varepsilon,-v) .
$$

Pour le cas $n$ pair, il faut appliquer la théorie de Morse pour les variétés à bord. Un point critique $p_{i}$ de $x_{1 \mid C_{t}^{\varepsilon}}$ est à vecteur gradient rentrant pour $\left\{f_{t} \geq 0\right\}$ (resp. $\left.\left\{f_{t} \leq 0\right\}\right)$ si et seulement si $\frac{\partial f_{t}}{\partial x_{1}}\left(p_{i}\right)>0$ (resp. $\left.\frac{\partial f_{t}}{\partial x_{1}}\left(p_{i}\right)<0\right)$. Dans [Du], Théorème 2.5 , on considère une variété à bord $M$, une fonction de Morse $f: M \rightarrow \mathbb{R}$ et on exprime les caractéristiques d'Euler relatives $\chi(M \cap\{f \geq 0\}, M \cap\{f=0\})$ et $\chi(M \cap\{f \leq 0\}, M \cap\{f=0\})$ en fonction des indices des points critiques de $f$. En appliquant ce théorème et en ayant remarqué au préalable que $x_{1 \mid\left\{f_{t} \geq 0\right\} \cap B_{\varepsilon}}$ n'admet pas de points critiques à l'intérieur de $\left\{f_{t} \geq 0\right\} \cap B_{\varepsilon}$, on trouve :

$$
\begin{gathered}
\chi\left(\left\{f_{t} \geq 0\right\} \cap\left\{x_{1} \geq 0\right\} \cap B_{\varepsilon},\left\{f_{t} \geq 0\right\} \cap\left\{x_{1}=0\right\} \cap B_{\varepsilon}\right)=\sum_{\substack{x_{1}\left(p_{i}\right)>0 \\
\frac{\partial f_{t}}{\partial x_{1}}\left(p_{i}\right)>0}}(-1)^{\lambda_{i}} \\
\chi\left(\left\{f_{t} \geq 0\right\} \cap\left\{x_{1} \leq 0\right\} \cap B_{\varepsilon},\left\{f_{t} \geq 0\right\} \cap\left\{x_{1}=0\right\} \cap B_{\varepsilon}\right)=-\sum_{\substack{x_{1}\left(p_{i}\right)<0 \\
\frac{\partial f_{t}}{\partial x_{1}\left(p_{i}\right)<0}}}(-1)^{\lambda_{i}} .
\end{gathered}
$$


De même,

$$
\begin{aligned}
& \chi\left(\left\{f_{t} \leq 0\right\} \cap\left\{x_{1} \geq 0\right\} \cap B_{\varepsilon},\left\{f_{t} \leq 0\right\} \cap\left\{x_{1}=0\right\} \cap B_{\varepsilon}\right)=\sum_{\substack{x_{1}\left(p_{i}\right)>0 \\
\frac{\partial t_{t}}{\partial x_{1}}\left(p_{i}\right)<0}}(-1)^{\lambda_{i}}, \\
& \chi\left(\left\{f_{t} \leq 0\right\} \cap\left\{x_{1} \leq 0\right\} \cap B_{\varepsilon},\left\{f_{t} \leq 0\right\} \cap\left\{x_{1}=0\right\} \cap B_{\varepsilon}\right)=-\sum_{\substack{x_{1}\left(p_{i}\right)<0 \\
\frac{\partial f_{t}}{\partial x_{1}}\left(p_{i}\right)>0}}(-1)^{\lambda_{i}} .
\end{aligned}
$$

Il convient d'être prudent car $\left\{f_{t} \geq 0\right\} \cap B_{\varepsilon}$ et $\left\{f_{t} \leq 0\right\} \cap B_{\varepsilon}$ sont des variétés à coins. Il peut apparaître des points critiques sur $f_{t}^{-1}(0) \cap S_{\varepsilon},\left\{f_{t}>0\right\} \cap S_{\varepsilon}$ et $\left\{f_{t}<0\right\} \cap S_{\varepsilon}$. Il est facile de voir que le point critique de $x_{1}$ sur $\left\{x_{1} \geq 0\right\} \cap S_{\varepsilon}$ (resp. $\left\{x_{1} \leq 0\right\} \cap S_{\varepsilon}$ ) est à gradient sortant (resp. rentrant). De plus, le même argument que [Du] Lemma 4.1 montre que les gradients des points critiques de

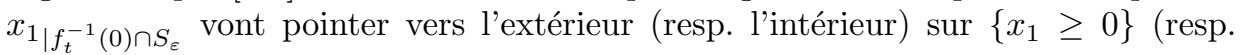
$\left.\left\{x_{1} \leq 0\right\}\right)$ et donc n'interviennent pas dans les formules ci-dessus. La combinaison $(4)+(5)-(6)-(7)$ donne

$$
\begin{gathered}
\chi\left(\left\{f_{t} \geq 0\right\} \cap\left\{x_{1} \geq 0\right\} \cap B_{\varepsilon}\right)+\chi\left(\left\{f_{t} \geq 0\right\} \cap\left\{x_{1} \leq 0\right\} \cap B_{\varepsilon}\right) \\
-2 \chi\left(\left\{f_{t} \geq 0\right\} \cap\left\{x_{1}=0\right\} \cap B_{\varepsilon}\right)-\chi\left(\left\{f_{t} \leq 0\right\} \cap\left\{x_{1} \geq 0\right\} \cap B_{\varepsilon}\right) \\
-\chi\left(\left\{f_{t} \leq 0\right\} \cap\left\{x_{1} \leq 0\right\} \cap B_{\varepsilon}\right)+2 \chi\left(\left\{f_{t} \leq 0\right\} \cap\left\{x_{1}=0\right\} \cap B_{\varepsilon}\right) \\
=\sum_{i} \text { signe } \frac{\partial f_{t}}{\partial x_{1}}\left(p_{i}\right)(-1)^{\lambda_{i}} .
\end{gathered}
$$

La suite de Mayer-Vietoris et la relation (3) permettent de conclure.

Théorème 5.2. Pour tout $v \in U$,

$$
\begin{aligned}
& \lim _{\varepsilon \rightarrow 0} \lim _{t \rightarrow 0^{+}}[I(t, \varepsilon, v)+I(t, \varepsilon,-v)]=-\operatorname{deg}_{0} \nabla F-\operatorname{deg}_{0} H+\operatorname{deg}_{0} \nabla\left(f_{\mid P_{v}=0}\right), \\
& \lim _{\varepsilon \rightarrow 0} \lim _{t \rightarrow 0^{-}}[I(t, \varepsilon, v)+I(t, \varepsilon,-v)]=-\operatorname{deg}_{0} \nabla F+\operatorname{deg}_{0} H+\operatorname{deg}_{0} \nabla\left(f_{\mid P_{v}=0}\right) .
\end{aligned}
$$

Preuve. C'est une combinaison du théorème précédent et du Théorème 3.2 .

Par le Théorème de Bezout, $|I(t, \varepsilon, v)+I(t, \varepsilon,-v)| \leq(\operatorname{deg} F) \cdot(\operatorname{deg} F-1)^{n-1}$. Le complémentaire de $U$ dans $S^{n-1}$ étant de mesure nulle, on peut appliquer le théorème de convergence dominée de Lebesgue à la fonction dépendant de $t$, $v \mapsto I(t, \varepsilon, v)+I(t, \varepsilon,-v)$, puis à la fonction dépendant de $\varepsilon, v \mapsto \lim _{t \rightarrow 0}[I(t, \varepsilon, v)+$ $I(t, \varepsilon,-v)]$. On obtient

$$
\begin{aligned}
& \lim _{\varepsilon \rightarrow 0} \lim _{t \rightarrow 0^{+}} \int_{S^{n-1}}[I(t, \varepsilon, v)+I(t, \varepsilon,-v)] d v= \\
& \quad \int_{S^{n-1}} \lim _{\varepsilon \rightarrow 0} \lim _{t \rightarrow 0^{+}}[I(t, \varepsilon, v)+I(t, \varepsilon,-v)] d v,
\end{aligned}
$$


et

$$
\begin{aligned}
\lim _{\varepsilon \rightarrow 0} \lim _{t \rightarrow 0^{-}} \int_{S^{n-1}}[I(t, \varepsilon, v)+I(t, \varepsilon,-v)] d v \\
\quad=\int_{S^{n-1}} \lim _{\varepsilon \rightarrow 0} \lim _{t \rightarrow 0^{-}}[I(t, \varepsilon, v)+I(t, \varepsilon,-v)] d v .
\end{aligned}
$$

D'après le Théorème 5.2 :

$$
\begin{aligned}
\lim _{\varepsilon \rightarrow 0} \lim _{t \rightarrow 0^{+}} \int_{S^{n-1}} & {[I(t, \varepsilon, v)+I(t, \varepsilon,-v)] d v } \\
& =-\operatorname{Vol}\left(S^{n-1}\right)\left[\operatorname{deg}_{0} \nabla F+\operatorname{deg}_{0} H\right]+\int_{S^{n-1}} \operatorname{deg}_{0} \nabla\left(f_{\mid P_{v}=0}\right) d v
\end{aligned}
$$

et

$$
\begin{aligned}
\lim _{\varepsilon \rightarrow 0} \lim _{t \rightarrow 0^{-}} \int_{S^{n-1}} & {[I(t, \varepsilon, v)+I(t, \varepsilon,-v)] d v } \\
& =-\operatorname{Vol}\left(S^{n-1}\right)\left[\operatorname{deg}_{0} \nabla F-\operatorname{deg}_{0} H\right]+\int_{S^{n-1}} \operatorname{deg}_{0} \nabla\left(f_{\mid P_{v}=0}\right) d v .
\end{aligned}
$$

Théorème 5.3.

$\lim _{\varepsilon \rightarrow 0} \lim _{t \rightarrow 0^{+}} \int_{C_{t}^{\varepsilon}} k d v_{t}=-\frac{1}{2} \operatorname{Vol}\left(S^{n-1}\right)\left[\operatorname{deg}_{0} \nabla F+\operatorname{deg}_{0} H\right]+\frac{1}{2} \int_{S^{n-1}} \operatorname{deg}_{0} \nabla\left(f_{\mid P_{v}=0}\right) d v$,
$\lim _{\varepsilon \rightarrow 0} \lim _{t \rightarrow 0^{-}} \int_{C_{t}^{\varepsilon}} k d v_{t}=-\frac{1}{2} \operatorname{Vol}\left(S^{n-1}\right)\left[\operatorname{deg}_{0} \nabla F-\operatorname{deg}_{0} H\right]+\frac{1}{2} \int_{S^{n-1}} \operatorname{deg}_{0} \nabla\left(f_{\mid P_{v}=0}\right) d v$.

Preuve. Soit $\Delta=\gamma_{t, \varepsilon}(\{x \mid k(x)=0\})$ l'ensemble des valeurs critiques de $\gamma_{t, \varepsilon}$. D'après le théorème de Sard, $\Delta$ est de mesure nulle et donc $\Delta \cup-\Delta$ l'est également ( $-\Delta$ désigne le symétrique de $\Delta$ par rapport à l'origine). L'ensemble $\gamma_{t, \varepsilon}^{-1}(\Delta \cup-\Delta) \backslash$ $\{x \mid k(x)=0\}$ est aussi de mesure nulle car c'est un ensemble de points réguliers de $\gamma_{t, \varepsilon}$ dont l'image est contenue dans un ensemble de mesure nulle. On peut écrire alors :

$$
\int_{C_{t}^{\varepsilon}} k d v_{t}=\int_{C_{t}^{\varepsilon} \backslash\{x \mid k(x)=0\}} \operatorname{Jac}\left(\gamma_{t, \varepsilon}\right) d v_{t}=\int_{C_{t}^{\varepsilon} \backslash \gamma_{t, \varepsilon}^{-1}(\Delta \cup-\Delta)} \operatorname{Jac}\left(\gamma_{t, \varepsilon}\right) d v_{t},
$$

où Jac désigne le jacobien. Pour tout $i \in \mathbb{Z}$, on définit $A_{i}$ par

$$
A_{i}=\left\{v \in S^{n-1} \backslash\{\Delta \cup-\Delta\} \mid I(t, \varepsilon, v)=i\right\}
$$

et on a

$$
\int_{C_{t}^{\varepsilon}} k d v_{t}=\sum_{i} \int_{\gamma_{t, \varepsilon}^{-1}\left(A_{i}\right)} \operatorname{Jac}\left(\gamma_{t, \varepsilon}\right) d v_{t}=\sum_{i} i \int_{A_{i}} d v .
$$

En décomposant $A_{i}$ en ses composantes connexes, $A_{i}=\sqcup A_{i k}$,

$$
\int_{C_{t}^{\varepsilon}} k d v_{t}=\sum_{i} \sum_{k} i \int_{A_{i k}} d v
$$


Si $A_{i k}=-A_{i k}$ alors pour tout $v \in A_{i k}, I(t, \varepsilon, v)=I(t, \varepsilon,-v)=i$ et $i=$ $\frac{1}{2}[I(t, \varepsilon, v)+I(t, \varepsilon,-v)]$. Si $A_{i k} \neq-A_{i k}$ alors il existe un couple $(j, l)$ tel que $A_{j l}=-A_{i k}$. On a $\int_{A_{i k}} d v=\int_{A_{j l}} d v$. Dans ce cas

$$
\begin{gathered}
i \int_{A_{i k}} d v+j \int_{A_{j l}} d v=\frac{1}{2}(i+j) \int_{A_{i k}} d v+\frac{1}{2}(i+j) \int_{A_{j l}} d v \\
=\frac{1}{2} \int_{A_{i k}}[I(t, \varepsilon, v)+I(t, \varepsilon,-v)] d v+\frac{1}{2} \int_{A_{j l}}[I(t, \varepsilon, v)+I(t, \varepsilon,-v)] d v .
\end{gathered}
$$

On en déduit la formule d'échange :

$$
\begin{aligned}
\int_{C_{t}^{\varepsilon}} k(x) d v_{t}=\frac{1}{2} \int_{S^{n-1} \backslash(\Delta \cup-\Delta)}[I(t, \varepsilon, v) & +I(t, \varepsilon,-v)] d v= \\
& \frac{1}{2} \int_{S^{n-1}}[I(t, \varepsilon, v)+I(t, \varepsilon,-v)] d v .
\end{aligned}
$$

Un passage à la limite permet de conclure.

\section{Corollaire 5.4.}

$$
\lim _{\varepsilon \rightarrow 0} \lim _{t \rightarrow 0^{+}} \int_{C_{t}^{\varepsilon}} k d v_{t}-\lim _{\varepsilon \rightarrow 0} \lim _{t \rightarrow 0^{-}} \int_{C_{t}^{\varepsilon}} k d v_{t}=-\operatorname{Vol}\left(S^{n-1}\right) \operatorname{deg}_{0} H .
$$

Corollaire 5.5. Si $f$ admet une singularité algébriquement isolée en 0 :

$$
\left|\lim _{\varepsilon \rightarrow 0} \lim _{t \rightarrow 0^{+}} \int_{C_{t}^{\varepsilon}} k d v_{t}\right| \leq \frac{1}{2} \operatorname{Vol}\left(S^{n-1}\right) M_{+},
$$

où

$$
\begin{aligned}
M_{+}=\operatorname{Max}\left(\left|\operatorname{deg}_{0} \nabla F+\operatorname{deg}_{0} H+\left(\mu^{n-1}\left(f_{\mathbb{C}}\right)\right)^{1-\frac{1}{n-1}}\right|,\right. \\
\\
\left.\left|\operatorname{deg}_{0} \nabla F+\operatorname{deg}_{0} H-\left(\mu^{n-1}\left(f_{\mathbb{C}}\right)\right)^{1-\frac{1}{n-1}}\right|\right),
\end{aligned}
$$

et

$$
\left|\lim _{\varepsilon \rightarrow 0} \lim _{t \rightarrow 0^{-}} \int_{C_{t}^{\varepsilon}} k d v_{t}\right| \leq \frac{1}{2} \operatorname{Vol}\left(S^{n-1}\right) M_{-},
$$

où

$$
\begin{aligned}
& M_{-}=\operatorname{Max}\left(\left|-\operatorname{deg}_{0} \nabla F+\operatorname{deg}_{0} H+\left(\mu^{n-1}\left(f_{\mathbb{C}}\right)\right)^{1-\frac{1}{n-1}}\right|,\right. \\
&\left.\left|-\operatorname{deg}_{0} \nabla F+\operatorname{deg}_{0} H-\left(\mu^{n-1}\left(f_{\mathbb{C}}\right)\right)^{1-\frac{1}{n-1}}\right|\right),
\end{aligned}
$$

$\mu^{n-1}\left(f_{\mathbb{C}}\right)$ étant le nombre de Milnor de la singularité obtenue en coupant $f_{\mathbb{C}}^{-1}(0)$ par un hyperplan générique. 
Preuve. D'après la Remarque 4.2, on peut choisir $U$ tel que pour tout $v \in U$, le complexifié de $f:\left\{P_{v}=0\right\} \rightarrow \mathbb{R}$ ait pour nombre de Milnor $\mu^{n-1}\left(f_{\mathbb{C}}\right)$. D'après le Théorème 2.1 de [EL], pour tout $v \in U$,

$$
\left|\operatorname{deg}_{0} \nabla\left(f_{\mid P_{v}=0}\right)\right| \leq\left(\mu^{n-1}\left(f_{\mathbb{C}}\right)\right)^{1-\frac{1}{n-1}} .
$$

Appliqué à la déformation $F(t, x)=f(x)-t$, le Théorème 5.3 s'écrit :

Théorème 5.6. Soit $f: \mathbb{R}^{n} \rightarrow \mathbb{R}$ un polynôme tel que $f(0)=0$ et tel que 0 soit un point critique isolé de $f$. Alors

$$
\begin{aligned}
& \lim _{\varepsilon \rightarrow 0} \lim _{\lambda \rightarrow 0^{+}} \int_{C_{\lambda}^{\varepsilon}} k d v_{\lambda}=\frac{1}{2} \operatorname{Vol}\left(S^{n-1}\right) \operatorname{deg}_{0} \nabla f+\frac{1}{2} \int_{S^{n-1}} \operatorname{deg}_{0} \nabla\left(f_{\mid P_{v}=0}\right) d v, \\
& \lim _{\varepsilon \rightarrow 0} \lim _{\lambda \rightarrow 0^{-}} \int_{C_{\lambda}^{\varepsilon}} k d v_{\lambda}=-\frac{1}{2} \operatorname{Vol}\left(S^{n-1}\right) \operatorname{deg}_{0} \nabla f+\frac{1}{2} \int_{S^{n-1}} \operatorname{deg}_{0} \nabla\left(f_{\mid P_{v}=0}\right) d v,
\end{aligned}
$$

où $C_{\lambda}^{\varepsilon}$ est la fibre $f^{-1}(\lambda) \cap B_{\varepsilon}^{n}$.

Nous présentons ici un premier exemple. Soit $f(x, y, z)=x^{2}+y^{3}-z x$ et soit $F(t, x, y, z)=x^{2}+y^{3}-z x+t(x+y+z)$. A l'aide du programme de Lecki mentionné dans l'introduction, on trouve $\operatorname{deg}_{0} \nabla F=1$ et $\operatorname{deg}_{0} H=-1$. Il nous faut calculer $\int_{S^{2}} \operatorname{deg}_{0} \nabla\left(f_{\mid P_{v}=0}\right) d v$. Soit $v=(a, b, c) \in S^{2}$, alors $(x, y, z) \in\left\{P_{v}=0\right\}$ si et seulement si $a x+b y+c z=0$. Si $c \neq 0$ alors

$$
f_{\mid P_{v}=0}(x, y, z)=f\left(x, y,-\frac{a}{c} x-\frac{b}{c} y\right)=\left(1+\frac{a}{c}\right)^{2} x^{2}+\frac{b}{c} x y+y^{3}:=g(x, y) .
$$

On a $\frac{\partial g}{\partial x}(x, y)=2\left(1+\frac{a}{c}\right) x+\frac{b}{c} y$ et $\frac{\partial g}{\partial y}(x, y)=3 y^{2}+\frac{b}{c} x$. Il est facile de voir que $(0,0)$ est un zéro isolé de $\nabla g$. De plus

$$
\left|\begin{array}{cc}
\frac{\partial^{2} g}{\partial^{2} x}(0,0) & \frac{\partial^{2} g}{\partial x \partial y}(0,0) \\
\frac{\partial^{2} g}{\partial x \partial y}(0,0) & \frac{\partial^{2} g}{\partial^{2} y}(0,0)
\end{array}\right|=-\frac{b^{2}}{c^{2}}
$$

et donc, pour $b$ et $c$ différents de $0,(0,0)$ est un zéro non-dégénéré de $\nabla g$ et $\operatorname{deg}_{0} \nabla g=-1$. On trouve que

$$
\int_{S^{2}} \operatorname{deg}_{0} \nabla\left(f_{\mid P_{v}=0}\right) d v=-4 \pi
$$

et que

$$
\lim _{\varepsilon \rightarrow 0} \lim _{t \rightarrow 0^{+}} \int_{C_{t}^{\varepsilon}} k d v_{t}=-2 \pi
$$

et $\lim _{\varepsilon \rightarrow 0} \lim _{t \rightarrow 0^{-}} \int_{C_{t}^{\varepsilon}} k d v_{t}=-6 \pi$. 


\section{Le cas des courbes planes}

Soit $f: \mathbb{R}^{2} \rightarrow \mathbb{R}$ un polynôme tel que $f(0)=0$ et $\nabla f^{-1}(0) \cap B_{\varepsilon} \subset\{0\}$ pour $0<\varepsilon \ll 1$. Soit $e$ la multiplicité de $f$, c'est-à-dire que $f$ s'écrit :

$$
f(x, y)=f_{e}(x, y)+f_{e+1}(x, y)+\cdots+f_{d}(x, y),
$$

où $d$ est le degré de $f$ et où pour tout $i \in\{e, \ldots, d\}, f_{i}$ est la composante homogène de degré $i$ de $f$. Le Théorème 5.3 devient alors :

Théorème 6.1. Si e est impair,

$$
\begin{aligned}
& \lim _{\varepsilon \rightarrow 0} \lim _{t \rightarrow 0^{+}} \int_{C_{t}^{\varepsilon}} k d v_{t}=-\pi \cdot\left(\operatorname{deg}_{0} \nabla F+\operatorname{deg}_{0} H\right), \\
& \lim _{\varepsilon \rightarrow 0} \lim _{t \rightarrow 0^{-}} \int_{C_{t}^{\varepsilon}} k d v_{t}=-\pi \cdot\left(\operatorname{deg}_{0} \nabla F-\operatorname{deg}_{0} H\right) .
\end{aligned}
$$

Si e est pair,

$$
\begin{aligned}
& \lim _{\varepsilon \rightarrow 0} \lim _{t \rightarrow 0^{+}} \int_{C_{t}^{\varepsilon}} k d v_{t}=-\pi \cdot\left(\operatorname{deg}_{0} \nabla F+\operatorname{deg}_{0} H\right)+\int_{-\frac{\pi}{2}}^{\frac{\pi}{2}} \text { signe } f_{e}(-\tan \theta, 1) d \theta, \\
& \lim _{\varepsilon \rightarrow 0} \lim _{t \rightarrow 0^{-}} \int_{C_{t}^{\varepsilon}} k d v_{t}=-\pi \cdot\left(\operatorname{deg}_{0} \nabla F-\operatorname{deg}_{0} H\right)+\int_{-\frac{\pi}{2}}^{\frac{\pi}{2}} \operatorname{signe} f_{e}(-\tan \theta, 1) d \theta .
\end{aligned}
$$

Preuve. On remarque d'abord que si $g:(\mathbb{R}, 0) \rightarrow(\mathbb{R}, 0)$ est un germe analytique de multiplicité $\epsilon$, alors $\operatorname{deg}_{0} g=0$ si $\epsilon$ est pair et $\operatorname{deg}_{0} g=$ signe $g_{\epsilon}(1)$ si $\epsilon$ est impair.

Soit $v \in S^{1}$, il existe $\theta \in[-\pi, \pi]$ tel que $v=(\cos \theta, \sin \theta)$. Dans ce cas, $(x, y) \in$ $\left\{P_{v}=0\right\}$ si et seulement si $x \cos \theta+y \sin \theta=0$. Si $\theta \neq \pm \frac{\pi}{2}$, on a alors

$$
f_{\mid P_{v}=0}(x, y)=f(-\tan \theta y, y)=\sum_{i=e}^{d} y^{i} f_{i}(-\tan \theta, 1),
$$

et donc

$$
\nabla\left(f_{\mid P_{v}=0}\right)(x, y)=\sum_{i=e}^{d} i y^{i-1} f_{i}(-\tan \theta, 1) .
$$

Si $e$ est impair, $\operatorname{deg}_{0} \nabla\left(f_{\mid P_{v}=0}\right)=0$. Si $e$ est pair, $\operatorname{deg}_{0} \nabla\left(f_{\mid P_{v}=0}\right)=$ signe $f_{e}(-\tan \theta, 1)$. On trouve pour ce dernier cas :

$$
\begin{gathered}
\int_{S^{1}} \operatorname{deg}_{0} \nabla\left(f_{\mid H_{v}=0}\right) d v=\int_{-\pi}^{\pi} \text { signe } f_{e}(-\tan \theta, 1) d \theta \\
=2 \int_{-\frac{\pi}{2}}^{\frac{\pi}{2}} \text { signe } f_{e}(-\tan \theta, 1) d \theta .
\end{gathered}
$$


Exemple. Soit $f(x, y)=y^{2}-x^{3}$ et soit $F(t, x, y)=y^{2}-x^{3}-t x$. A l'aide du programme, on trouve $\operatorname{deg}_{0} \nabla F=-1$ et $\operatorname{deg}_{0} H=1$. La multiplicité de $f$ est 2 et $f_{2}(x, y)=x^{2}$. On a donc

$$
\int_{-\frac{\pi}{2}}^{\frac{\pi}{2}} \text { signe } f_{2}(-\tan \theta, 1) d \theta=\pi .
$$

Finalement, on obtient

$$
\lim _{\varepsilon \rightarrow 0} \lim _{t \rightarrow 0^{+}} \int_{C_{t}^{\varepsilon}} k d v_{t}=\pi \text { et } \lim _{\varepsilon \rightarrow 0} \lim _{t \rightarrow 0^{-}} \int_{C_{t}^{\varepsilon}} k d v_{t}=3 \pi .
$$

Cette différence entre les deux limites est due au fait que la fibre $f_{t}^{-1}(0), t<0$, a un ovale de plus que la fibre $f_{t}^{-1}(0), t>0$.

\section{Références}

[Ar] V. I. Arnold, Index of a singular point of a vector field, the Petrovski-Oleinik inequality, and mixed Hodge structures, Funct. Anal. and its Appli. 12 (1978), 1-14.

[BCR] J. Bochnak, M. Coste and M. F. Roy, Géométrie algébrique réelle, Ergebnisse der Mathematik 12 Springer-Verlag, 1987.

[Du] N. Dutertre, Degree formulas for a topological invariant of bifurcations of function-germs, Kodai Math. Journal 23, No. 3 (2000), 442-461.

[EL] D. Eisenbud and H. I. Levine, An algebraic formula for the degree of a $C^{\infty}$ map-germ, Annals of Mathematics 106 (1977), 19-44.

[Fu1] T. Fukui, An algebraic formula for a topological invariant of bifurcation of 1-parameter family of function-germs, in : Stratifications, singularities, and differential equations, II (Marseille, 1990; Honolulu, HI, 1990), 45-54, Travaux en cours 55, Hermann, Paris, 1997.

[Fu2] T. Fukui, Mapping degree formula for 2-parameter bifurcation of function-germs, Topology 32 (1993), 567-571.

[Kh] G. M. Khimshiashvili, On the local degree of a smooth map, Soobshch. Akad. Nauk Gruz. SSR 85 (1977), 309-311.

[La] R. Langevin, Courbure et singularités complexes, Comment. Math. Helvetici 54 (1979), $6-16$.

[LS] A. Lecki and Z. Szafraniec, Applications of the Eisenbud-Levine's theorem to real algebraic geometry, Computational Algebraic Geometry, 177-184, Progress in Mathematics 109, Birkhäuser, 1993.

[Ri] J.-J. Risler, On the curvature of the Milnor fiber, Préprint.

[Sz] Z. Szafraniec, A formula for the Euler characteristic of a real algebraic manifold, manuscripta mathematica 85 (1994), 345-360.

[Te] B. Teissier, Introduction to equisingularity problems, Proc. Symp. in Pure Math. 29 Arcata 1974, Amer. Math. Soc. (1975), 593-632.

[Wa] C. T. C. Wall, Topological invariance of the Milnor number mod 2, Topology 22 (1983), $345-350$. 
Nicolas Dutertre

Centre de Recerca Matemàtica

Institut d'Estudis Catalans

Apartat 50

E-08193 Bellaterra

España

e-mail : dutertre@crm.es

(Received: January 26, 2001)

To access this journal online:

(4) http://www.birkhauser.ch 